\title{
Bright Light Treatment of Depression for 28 Adolescents
}

\author{
Helmut Niederhofer ${ }^{1 *}$, Kai von Klitzing ${ }^{2}$ \\ ${ }^{1}$ Saechsisches Krankenhaus Rodewisch, Child and Adolescent Psychiatry, Rodewisch, Germany; ${ }^{2}$ Child and Adolescent Psychiatry, \\ University of Leipzig, Leipzig, Germany. \\ E-mail: *helmutniederhofer@yahoo.de, kai.vonklitzing@uniklinik-leipzig.de
}

Received September $24^{\text {th }}, 2011$; revised November $9^{\text {th }}$, 2011; accepted November $17^{\text {th }}, 2011$.

\begin{abstract}
Background: Bright light therapy, an effective therapeutic option for depressive adults, could provide safe, economic, and effective rapid recovery also in adolescents. Method: This was a randomized cross-over trial, i.e. that 14 patients received first dim white light (50 lux) one hour a day for one week and then Bright light therapy (2500 Lux) for one week. 14 patients received first Bright light therapy and then dim white light. For assessment of depressive symptoms, Beck depression inventory scales were administered 1 week before and one day before dim white light treatment, on the day between dim white light and bright white light treatment, on the day after bright white light treatment and one week after bright white light treatment. Saliva melatonin and cortisol samples were collected at 08:00 a.m. and 08:00 p.m., 1 week before and one day before dim white light treatment, on the day between dim white light and bright white light treatment, on the day after bright white light treatment and one week after bright white light treatment and assayed for melatonin and cortisol to observe any change in circadian timing. Results: 28 volunteers, between 14 and 17 years old, completed the study. Beck depression inventory scores improved significantly. The assays of morning saliva melatonin showed significant differences between Bright white light and dim white light $(p=0.005)$, those of evening saliva melatonin and saliva cortisol did not show significant differences. No significant adverse reactions were observed. Conclusion: Antidepressant response to bright light treatment in this age group was statistically superior to dim white light.
\end{abstract}

Keywords: Bright Light Therapy, Non-Seasonal Depression, Adolescents

\section{Introduction}

Antidepressants are effective treating psychiatric disorders in adolescents, but nevertheless depression is often treated inadequately. In adolescents, there is a greater risk of medication side effects. These difficulties suggest the establishment of additional biological treatment options for depressed adolescents either to supplement or replace pharmacotherapy. Development of e.g. Bright light treatment, applied by a bright light box (2500 10,000 lux), in front of which patients are asked to sit for 60 - 120 minutes, preferably in the morning, could have benefit for adolescents with depression.

Bright light treatment for seasonal depression (SAD) has become accepted in the Clinical Practice Guidelines issued by the U.S. Department of Health and Human Services [1] and the American Psychiatric Association's Treatment of Psychiatric Disorders [2], because it is effective, rapid, safe, and inexpensive. Some light treatment study have been conducted to investigate nonsea- sonal major depressions, demonstrating that light is effective also for nonseasonal depression and improves also the circadian rhythm [3-5]. In nonseasonal depression, light treatment may produce more benefit when coadministered with antidepressant drugs [6].

There is some evidence that treatment with morning light is superior to evening light for SAD [7-10], but some studies have found little difference between timings $[11,12]$. The advantage of morning light could be partly explained by an anomalous order effect in cross-over designs [13]. Another explanation for the special effect of morning light could be, that it might work by suppressing or phase-advancing an overly-late melatonin offset $[14,15]$. Furthermore, conditions in which mood complaints are most prominent might have a common etiology in circadian phase malsynchronization which is characterized by abnormal entrainment of circadian rhythms to the solar day and/or abnormal relationships among rhythms in the body. 
The work of Neumeister et al. [16], Loving [17], and Bloching [18] suggests that bright light in combination with partial sleep deprivation produces remarkable antidepressant responses, as confirmed by dramatic contrasts between bright light and dim white light.

This paper reports a clinical trial of bright light treatment in 28 adolescents. It is based on a case series, where first preliminary results showed, that BLT is superior to dim white light, regarding depression scores and saliva melatonin levels [19]. This randomized trial was performed to demonstrate greater improvements in mood and sleep among volunteers receiving 1 week bright light as contrasted to dim white light.

\section{Methods}

28 Volunteers, 18 girls and 10 boys, aged 14 - 17 years, IQ 96 - 105, with significant depressive complaints, were evaluated for the clinical trial. Depressive symptoms existed for at least six months without any significant variation. Meeting full DSM-IV criteria for current major depressive disorder was not required, because many adolescents are significantly troubled by minor depressive disorders without meeting criteria for major depressive disorder. The DSM-IV criteria of seasonal depression applied: participants were recruited and studied in the autumn/winter/spring period. Participants did not receive any specific antidepressive medication, neither before nor during the study, but in summertime they reported significant remission for the last years. This fact might be due to school holidays in summertime, but anyway, the criteria for non-seasonal depression, especially because of the recruitment and study period, were not achieved. For that reason, seasonal depression must be diagnosed. This is important, because Bright light Therapy is proven to be effective, especially for seasonal [2], but also for non-seasonal depressive disorders in adults [6] and in adolescents [20]. Comorbidities have been defined as exclusion criteria. Informed consent was obtained from each participant, in accordance with the guidelines set forth by the Declaration of Helsinki. The local ethic commitee approved the study.

Depressive symptoms were assessed weekly by the Beck depression inventory [21]. The Beck depression inventory is a self-report questionnaire, which consists of 21 items, asking for symptoms such as e.g. sadness, suicidality, agitation and sleeping disorders, scoring from $0=$ not existent to $3=$ always existing. Beck depression inventory has an excellent variation sensitivity and $61 \%$ specificity for DSM-IV depression. A cut-off of 9 indicates, that scores of $>9$ are typical for depressive disorders. All participants had an initial Beck depression inventory score of $>9$.

Saliva samples of Melatonin and Cortisol (ELISA,
Immuno Biological Laboratories, Hamburg, Germany) were collected one week before and one day before dim white light treatment, on the day between dim white light and bright white light treatment, on the day after bright white light treatment and one week after bright white light treatment, at each time at 08:00 a.m. and 08:00 p.m., and assayed for melatonin and cortisol to characterize the circadian phase of the subject's saliva melatonin levels.

Furthermore, the volunteers completed a weekly Assessment for Treatment Emergent Events (SAFTEE) symptom scale [22]. This physical symptom inventory consists of 20 items (range: severe-moderate-mildminimal-not existing) and examines adverse reactions. In our trial, there were weekly symptom assessments. The investigators visited subjects weekly to assure their safety and their compliance with the study, and to administer and collect rating forms.

Any lifetime history of mania required exclusion of the potential volunteer, as a history of mania appears to predict a greatly increased risk of a manic switch during bright light treatment [5].

Patients were encouraged to continue ongoing psychotherapeutic treatment during the study, with the assumption that psychotherapy effects over an interval of 5 weeks were likely to be small, since there was no change of frequency of psychotherapy (2 sessions/week) since one month before the 4 week study period.

This was a randomized cross-over trial. After the baseline week, one half of the volunteers began dim white light treatment (dim white light) for one week followed by one week of BLT. The second half received, after the initial baseline week, first BLT for one week and then dim white light for another week.

The study protocol is depicted in Table 1.

Statistics were computed by SPSS V 17.0. We used Wilks Lambda test and for comparisons of bright white light/dim white light with the pre/post treatment period one factor analysis of variance for repeated measures with Bonferroni correction.

\section{Results}

The results of comparisons between bright white light/ dim white light and the pre/post treatment period are depicted in Table 2. Wilks Lambda test suggested the existence of significant differences of all scores and levels within the treatment period. One factor analysis of variance for repeated measures (Boferoni-corrected) shows, that most significant differences are mainly between bright white light and the pre/post treatment period. Interestingly also some significant differences between dim white light and the pre treatment (with respect to melatonin evening levels also with the post treatment period) period could be observed. 
Table 1. Study protocol.

Step 1: Volunteers began with the initial baseline week of the study. The purpose of the baseline week was to be able to differentiate changes of the Beck depression inventory score related to depression to that of dim white light and Bright light treatment.

Step 2: In the second week of the study, we asked the volunteers to sit in front of the dim white light light box (dim white light, 50 lux) for 60 minutes in the morning, from 09:00 to 10:00 a.m, Patients were allowed to play or listen to a story. If a Beck depression inventory score dropped $50 \%$ or more from the first day to the last day of the baseline week, the volunteer would have been dropped. This aspect of the design followed the general principle of clinical trial design that it is easier to obtain contrasts between active and dim white light treatments if early dim white light-responders are eliminated.

Step 3: In the third week of the study, we asked patients again to sit in front of the bright light box (2500 lux) for 60 minutes in the morning, i.e. from 09:00 to 10:00 a.m, i.e. for only one hour, because dosages of drugs and hence also Bright light treatment time should be reduced in children, adolescents, and older adults. In this time, patients played or listened to a story.

Step 4: In the fourth week of the study, patients receiced neither dim white light nor Bright white light. The purposes of this week of the study was again to be able to differentiate changes of the Beck depression inventory score related to depression to that of dim white light and Bright light treatment.

Table 2. Variations of Beck depression inventory, saliva melatonin morning and evening and saliva cortisol levels.

\begin{tabular}{|c|c|c|c|c|c|c|c|c|c|}
\hline & \multicolumn{2}{|c|}{ Wilks Lambda } & \multirow[t]{2}{*}{$\begin{array}{c}1 \text { Factor } \\
1 \text { week before }\end{array}$} & \multicolumn{3}{|c|}{$\begin{array}{c}\text { Analyasis of Variance, } \\
\text { Differences between bright white } \\
\text { light and... }\end{array}$} & \multirow[t]{2}{*}{$\begin{array}{l}\text { corrected by } \\
1 \text { week before }\end{array}$} & \multicolumn{2}{|c|}{$\begin{array}{l}\text { Bonferoni } \\
\text { Differences between dim } \\
\text { white light and... }\end{array}$} \\
\hline & & & & start & dim white light & post & & start & post \\
\hline & $\mathrm{F}$ & $\mathrm{p}$ & $\mathrm{p}$ & $\mathrm{p}$ & $\mathrm{p}$ & $\mathrm{p}$ & $\mathrm{p}$ & $\mathrm{p}$ & $\mathrm{p}$ \\
\hline Beck Depression Inventory & 3.91 & 0.014 & n.s. & 0.003 & n.s. & n.s. & n.s. & 0.017 & n.s. \\
\hline Melatonin morning & 5.64 & 0.03 & 0.006 & 0.018 & 0.005 & n.s. & n.s. & n.s. & n.s. \\
\hline Melatonin evening & 3.35 & 0.04 & 0.01 & 0.013 & n.s. & 0.007 & 0.018 & 0.024 & 0.003 \\
\hline Cortisol evening & 3.37 & n.s. & n.s. & n.s. & n.s. & n.s. & n.s. & n.s. & n.s. \\
\hline
\end{tabular}

Beck depression inventory scores were stable in the pre-treatment period, improved in group 1 during treatment significantly with dim white light, and then during treatment with bright light and rose again after the following week. Group 2 dropped from an initial score equal to that of group 1 significantly during BLT and rose again during dim white light treatment. In the posttreatment period, the score rose in both groups again up to the initial values (Figure 1).

In both groups, morning salivary melatonin was higher than in healthy controls (range: $<5 \mathrm{pg} / \mathrm{ml}$ ). Measured in the morning, it did not show significant variations in the pre-treatment and in the dim white light period, but there was a significant decrease in both groups in the BLT period. In the post-treatment period, we observed levels which did not differ significantly from those of the pretreatment period. Also in both groups, salivary melatonin, measured in the evening, was stable, but much lower than in healthy controls, (range $<10 \mathrm{pg} / \mathrm{ml}$ ) in the pretreatment period, and increased significantly after dim white light and again after BLT, and decreased again in the post-treatment week and finally did not differ significantly from the values of the pre-treatment period (Figure 2).
Regarding the salivary cortisol, we could not detect significant changes. The levels varied between 0.05 and $0.14 \mathrm{pg} / \mathrm{ml}$, without any significant changes between bright white light and dim white light.

With respect to the SAFTEE, the subscale "Depression" was scored exclusively by means of the Beck depression inventory. The symptom headache improved with bright light in four patients, change in appetite in two patients, insomnia in nine patients, fatigue in five patients and somnolence by one patient from mild to minimal. This improvement lasted until the end of the study. With respect to the other symptoms, patients did not report any change, i.e. they were always scored as "minimal" or "not existing", without any changes in the four weeks.

\section{Discussion}

Especially with respect to morning saliva melatonin levels, bright light treatment was superior to dim white light light. Also dim white light showed significant improvements (Beck depression inventory, evening saliva melatonin), but less than bright light treatment. The improvements found in the study are similar to recent investigations $[4,23]$ and might be attributed to several 


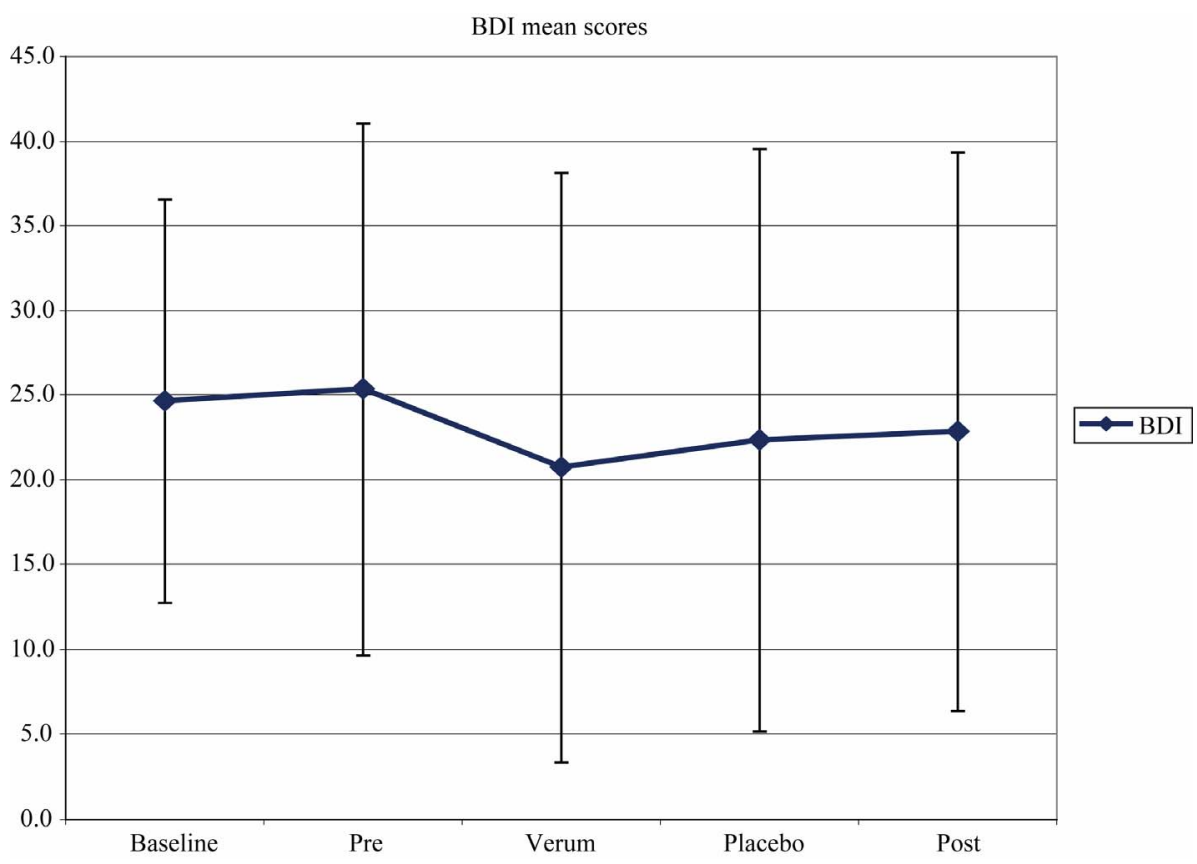

Figure 1. Variations of Beck depression inventory scores. Both subsamples have been summarized to improve presentation of contrasts between Bright white light and dim white light.

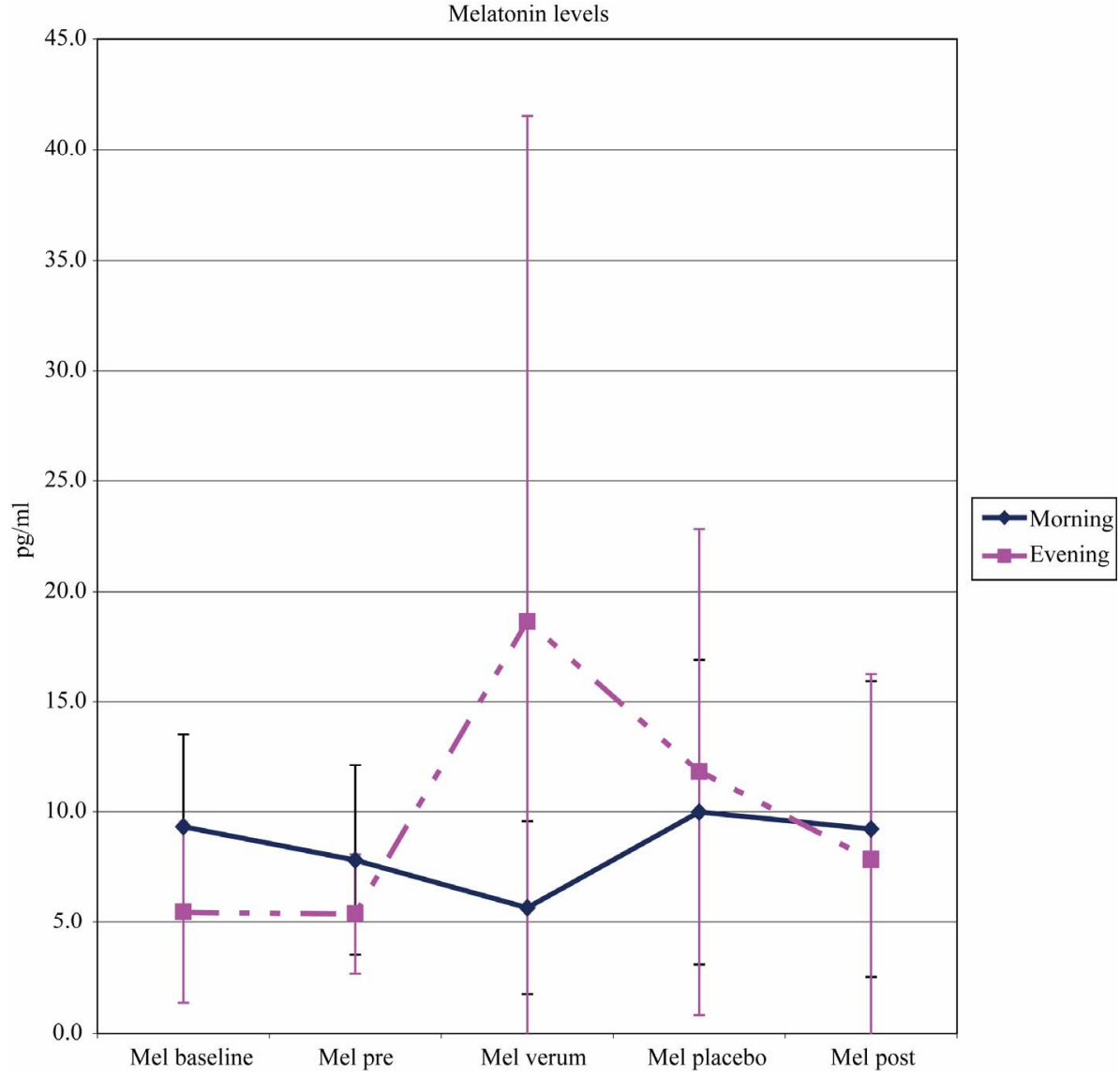

Figure 2. Variations of saliva melatonin levels $(\mathrm{pg} / \mathrm{ml})$. Both subsamples have been summarized to improve presentation of contrasts between Bright white light and dim white light. 
factors that were common to the treatment and control groups. Positive expectations, positive staff contacts, spontaneous remission and also the "dim white light" effect may have contributed to positive responses. The social structure and regularized sleep might also have been beneficial. An hour a day engaging in a treatment could have induced a reduction in depressive symptoms. A longer treatment period may be needed for this population with depressive symptoms to show more signifycant contrasts.

The antidepressant response to dim white light light treatment in this study is comparable to that reported for dim white light in drug studies [24,25]. However, dim white light response is significant and should be considered as a confound also in antidepressant light treatment studies. Exclusion of bipolar participants was necessary to prevent hypomanic adverse events, but possibly type 1 bipolar depressives are more light responsive, especially with respect to the circadian rhythm [5]. Compared to healthy controls, saliva melatonin evening levels, sleep, and activity all peak later in depressed patients. However, since increased light exposure is generally associated with more advanced melatonin evening peaks (as was observed comparing the bright light and dim white light period in this study), because bright white light treatment suppresses pineal melatonin synthesis and secretion [26], the results are consistent with the possibility that these depressed patients were subsensitive to circadian effects of light.

\section{Acknowledgements}

We wish to thank Mr. Joerg Domurath for his statistical support and the volunteers for participation.

\section{REFERENCES}

[1] Depression Guideline Panel, "Depression in Primary Care: Treatment of Major Depression," Vol. 2, Agency for Health Care Policy and Research, Washington DC, HHS, AHCPR Publication No. 93-0551, U.S. Government Printing Office, 1993.

[2] N. E. Rosenthal, “Light Therapy,” In: G. O. Gabbard, Ed., Treatment of Psychiatric Disorders, Vol. 1, American Psychiatric Press, Washington DC, 1995, pp. 1263-1273.

[3] A. Tuunainen, D. F. Kripke and T. Endo, "Light Therapy for Non-Seasonal Depression (Cochrane Review)," The Cochrane Library, 2004.

[4] R. Lieverse, E. J. Van someren, M. M. Nielen, B. M. uitdehaag, J. H. Smith and W. J. Hoogendijk, "Bright Light Treatment in Elderly Patients with Nonseasonal Major Depressive Disorder: A Randomized PlaceboControlled Trial," Archives of General Psychiatry, Vol. 68, No. 1, 2011, pp. 61-70. doi:10.1001/archgenpsychiatry.2010.183

[5] F. Benedetti, S. Dallaspezia, M. C. Fulgosi, B. Barbini, C.
Colombo and E. Smeraldi, "Phase Advance is an Arithmetic Coorelate of Antidepressant Response to Sleep Deprivation and Light Therapy in Bipolar Depression," Chronobiology International, Vol. 24, No. 5, 2007, pp. 921-937. doi:10.1080/07420520701649455

[6] A. J. Lewy, R. L. Sack, C. M. Singer, D. M. White and T. M. Hoban, "Winter Depression and the Phase-Shift Hypothesis for Bright Light'S Therapeutic Effects: History, Theory, and Experimental Evidence," Journal of Biological Rhythms, Vol. 3, No. 2, 1988, pp. 121-134. doi:10.1177/074873048800300203

[7] R. L. Sack, A. J. Lewy, D. M. White, C. M. Singer, M. J. Fireman and R. Vandiver, "Morning vs Evening Light Treatment for Winter Depression,” Archives of General Psychiatry, Vol. 47, No. 4, 1990, pp. 343-351.

[8] D. H. Avery, A. Khan, S. R. Dager, S. A. Cohen, G. B. Cox and D. L. Dunner, "Morning or Evening Bright Light Treatment of Winter Depression? The Significance of Hypersomnia,” Biological Psychiatry, Vol. 29, No. 2, 1991, pp. 117-126. doi:10.1016/0006-3223(91)90040-S

[9] J. S. Terman, M. Terman, E. S. Lo and T. B. Cooper, "Circadian Time of Morning Light Administration and Therapeutic Response in Winter Depression,” Archives of General Psychiatry, Vol. 58, No. 1, 2001, pp. 69-75. doi:10.1001/archpsyc.58.1.69

[10] M. Terman, J. S. Terman, F. M. Quitkin, P. J. McGrath, J. W. Stewart and B. Rafferty, "Light Therapy for Seasonal Affective Disorder: A Review of Efficacy," Neuropsychopharmacology, Vol. 2, 1989, pp. 1-22. doi:10.1016/0893-133X(89)90002-X

[11] M. Terman and J. S. Terman, "Randomized, Controlled Trial of Bright Light, Dawn Simulation and Negative Air Ionization for Winter Depression [Abstract]," Society for Light Treatment and Biological Rhythms, Vol. 14, 2002, p. 25.

[12] M. Terman, "Problems and Prospects for Use of Bright Light as a Therapeutic Intervention,” In: L. Wetterberg, Ed. Light and Biological Rhythms in Man. 1. Pergamon Press, Stockholm, 1993, pp. 421-436.

[13] A. Tuunainen, D. F. Kripke, J. A. Elliott, J. D. Assmus, K. M. Rex, M. R. Klauber and R. D. Langer, "Depression and Endogenous Melatonin in Postmenopausal Women," Journal of Affective Disorders, Vol. 69, No. 1, 2002; 149-158. doi:10.1016/S0165-0327(01)00303-2

[14] T. A. Wehr, W. C. Duncan, L. Sher, D. Aeschbach, P. J. Schwartz, E. H. Turner, T. T. Postolache and N. E. Rosenthal, "A Circadian Signal of Change of Season in Patients with Seasonal Affective Disorder," Archives of General Psychiatry, Vol. 58, No. 12, 2001, pp. 11081114. doi:10.1001/archpsyc.58.12.1108

[15] L. K. Sekula, J. F. Lucke, K. Heist, K. Czambel and R. T. Rubin, "Neuroendocrine Aspects of Primary Endogenous Depression XV: Mathematical Modeling of Nocturnal Melatonin Secretion in Major Depressives and Normal Controls,” Psychiatry Research, Vol. 69, No. 2-3, 1997, pp. 143-153. doi:10.1016/S0165-1781(96)02937-X

[16] A. Neumeister, R. Goessler, M. Lucht, T. Kapitany, C. 
Bamas and S. Kasper, "Bright Light Therapy Stabilizes the Antidepressant Effect of Partial Sleep Deprivation," Biological Psychiatry, Vol. 39, No. 1, 1996, pp. 16-21. doi:10.1016/0006-3223(95)00086-0

[17] R. T. Loving, D. F. Kripke, J. A. Eliott, N. C. Knickerbocker and M. A. Grandner, "Bright Light Treatment of Depression for Older Adults,” BMC Psychiatry, Vol. 9, 2005, p. 41. doi:10.1186/1471-244X-5-41

[18] B. Bloching, C. Dechene and K. L. Taschner, “Outlasting Antidepressant Effect of Late Partial Sleep Deprivation by Bright Light Therapy,” Journal of Sleep Research, Vol. 9, 2000, p. 21.

[19] H. Niederhofer and K. Klitzing, “Therapy Augmentation by Bright Light Treatment for Non-Seasonal Depression of Adolescents," Clinical Neuropsychiatry, Vol. 8, 2011, pp. 225-227

[20] H. Niederhofer and K. Klitzing, "Bright Light Treatment as mono-therapy of non-seasonal depression for 28 adolescents," International Journal of Psychiatry in Clinical Practice, Vol. 16, 2012, pp. 233-237

[21] A. T. Beck, C. H. Ward, M. Mendelson, J. Mock and J. Erbaugh, "An Inventory for Measuring Depression,” Ar- chives of General Psychiatry, Vol. 4, 1961, pp. 561-571.

[22] C. Moynihan, "Instruction Manual for Systematic Assessment for Treatment Emergent Events (SAFTEE)," ADAMHA, Rockville, MD, 1983.

[23] A. Wirz-Justice, F. Benedetti, M. Berger, R. W. Lam, K. Martiny, M. Terman and J. C. Wu, "Chronotherapeutics (Light and Wake Therapy) in Affective Disorders," Psychological Medicine, Vol. 35, No. 7, 2005, pp. 939944. doi:10.1017/S003329170500437X

[24] A. Khan, H. A. Warner and W. A. Brown, "Symptom Reduction and Suicide Risk in Patients Treated with Placebo in Antidepressant Clinical Trials," Archives of General Psychiatry, Vol. 57, No. 4, 2000, pp. 311-328. doi:10.1001/archpsyc.57.4.311

[25] C. I. Eastman, "What the Placebo Literature Can Tell Us about Light Therapy for SAD," Psychopharmacology Bulletin, Vol. 26, 1990, pp. 495-504.

[26] G. C. Brainard, M. D. Rollag and J. P. Hanifin, "Photic Regulation of Melatonin in Humans: Ocular and Neural Signal Transduction,” Journal of Biological Rhythms, Vol. 12, No. 6, 1997, pp. 537-546. doi:10.1177/074873049701200608 